\title{
On the importance of heat and mass transfer coupling for the characterization of hygroscopic insulation materials
}

\author{
Perré, $\mathbf{P}^{\mathrm{a}}{ }^{\mathrm{ab}}$; Challansonnex, $\mathbf{A}^{\mathrm{a}}$ \\ ${ }^{a}$ LGPM, CentraleSupélec, Université Paris-Saclay, 91190 Gif-sur-Yvette, France \\ b LGPM, CentraleSupélec, Centre Européen de Biotechnologie et de Bioéconomie (CEBB), 51110 \\ Pomacle, France.
}

*E-mail of the corresponding author: patrick.perre@centralesupelec.fr

\begin{abstract}
The present work is focused on mass transfer characterization of hygroscopic materials used for insulation, such as Low Density Fibreboards. Due to their particular morphology, these panels present a very high mass diffusivity in the connected gaseous phase and a very low thermal conductivity. This combination of properties exacerbates the coupling between heat and mass transfer in transient state.

Based on experimental data obtained with an original set-up and relevant simulations performed using a comprehensive physical formulation, a throughout vision of this question is proposed in the present study. In particular, we emphasize on:

- The impressive change in core temperature in terms of magnitude and duration,

- The great impact of the internal temperature gradient, which slows down mass diffusion,

- The dramatic error on mass diffusivity value if the coupling is ignored.
\end{abstract}

Keywords: experiment; identification; modelling; $R H$ at back-face; transient state. 
On the importance of heat and mass transfer coupling for the characterization of hygroscopic insulation materials.

\section{Introduction}

The thermal performances of constructions are getting more and more challenging and designers need to model physical phenomena with great accuracy ${ }^{[1-3]}$. Among them, the strong influence of coupled heat and mass transfer on energy consumption is well established. This is especially true for renewable materials, which generally have a high moisture buffering effect. Consequently, Building Energy Simulations (BES) models must account for coupled heat and mass transfer, which gave rise to a strong demand for mass transfer characterization: mass diffusivity and sorption isotherms. In addition, due to side effects such as molecular relaxation and dual-scale effects, these materials must be characterized in transient state as these materials are always in transient-state in buildings.

However, the characterisation of hygroscopic materials is not simple as, due to the latent heat of vaporization, no mass transfer can occur without heat transfer. Consequently, the temperature field is not uniform in the sample, which induces gradients of saturated water vapour, well known to affect mass diffusion. The present work is focused on a particular family of building materials: LDF or other hygroscopic insulation materials. Due to their particular morphology, these materials present a very high mass diffusivity in the connected gaseous phase and a very low thermal conductivity. Together with the hygroscopicity, this combination of properties exacerbates the coupling between heat and mass transfer in transient state. This fact is carefully analysed in the present work, using a unique combination of experiments and simulations.

\section{Materials and Methods}

\subsection{Experimental set-up}

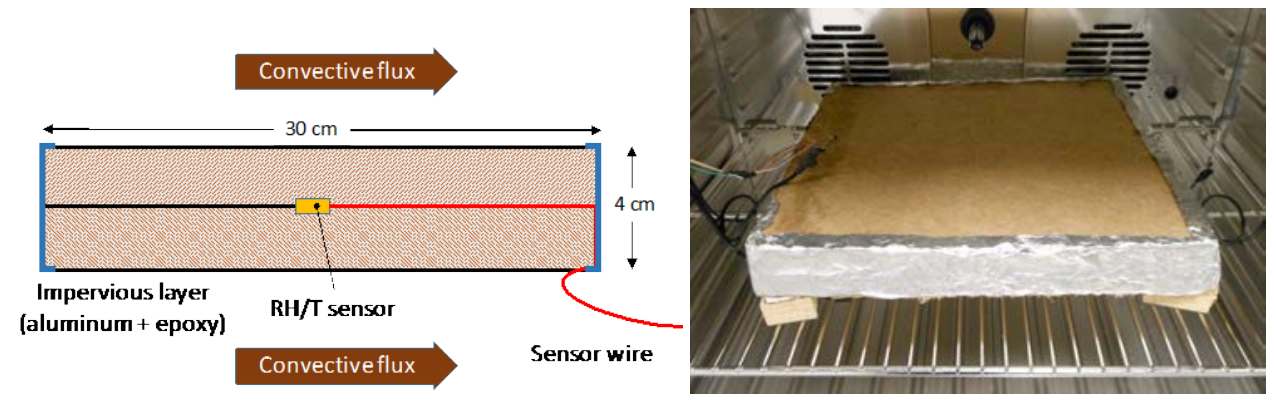

Fig. 1 - Schematic cross-sectional view of the double plate (left) and photograph of the sample placed inside the climatic chamber (right).

The material used in the present work is LDF $\left(160 \mathrm{~kg} \cdot \mathrm{m}^{-3}\right.$ low-density fiberboard produced by Steico, Therm). The experiment consists in submitting a sample face to a sudden variation of $\mathrm{RH}$ and to record the evolution of $\mathrm{RH}$ on the other face ${ }^{[4]}$. To rigorously assess the coupling between heat and mass transfer in this case of insulation material, a specific design was imagined to avoid any thermal perturbation and obtain 1-D transfer without any 
heat and mass flux at the back face of the sample (Fig. 1). For that purpose, a temperature/RH sensor (Sensirion SHT25, HDI Electronics, Perois, France) was placed between two $20-\mathrm{mm}$ thick plates of LDF. Side effects were avoided by choosing large dimensions $(30 \mathrm{~cm} \times 30 \mathrm{~cm}$ ), which is more than 7 times the diffusion thickness. Finally, to avoid lateral water vapor leakages, the two superimposed LDF plates were coated with an aluminium foil glued to the lateral faces by epoxy resin. This double sample is placed inside a climatic chamber (HPP110, Memmert, Schwabach, Germany) to control external conditions. Three RH/T sensors are placed inside the chamber to record the actual conditions applied to the sample.

\subsection{Results}

Fig. 2 presents a typical example of experimental data. This test was performed for a double layer of LDF panel ( $\rho=160 \mathrm{~kg} \cdot \mathrm{m}^{-3}$, thickness $=2 \times 20 \mathrm{~mm}$ ) for a stepwise change of RH from $20 \%$ to $40 \%$ at $35^{\circ} \mathrm{C}$. Low RH values were intentionally chosen to reduce the effect of molecular relaxation on the dynamic of sorption equilibrium ${ }^{[5]}$. The $\mathrm{RH}$ collected at the back-face of the sample needs about 10 hours to reach the new external RH. This information is the basic information used to identify the dimensionless mass diffusivity of the panel in the method proposed in ${ }^{[4]}$.

What is new in figure 2 is the temperature collected at the back-face of the sample. This value is relevant thanks to the symmetrical configuration: as the back-face of the sample is the value in the centre of two large plates, the assumption of 1-D transfer is valid and not artefact of heat transfer is likely to perturb the temperature value. As a result, we can observe an impressive temperature peak due to condensation: this peak is large in magnitude (more than $4^{\circ} \mathrm{C}$ above the ambiance) and in duration (more than 10 hours, the same duration as for mass transfer). Simulation performed using a comprehensive formulation of coupled heat and mass transfer will be used to analyse this impressive coupling and its effect on material characterisation.

\section{The physical formulation}

A comprehensive physical formulation was used in the present work. More detailed information regarding this set of equations can be found in published works ${ }^{[6]}$. For the sake of simplification, all liquid water contributions have been discarded, as the sample stays inside the hygroscopic domain. The simplified transport equations read as follows:

\section{Water conservation}

$$
\rho_{s} \frac{\partial X}{\partial t}+\nabla \cdot\left(\rho_{v} \bar{v}_{g}\right)=\nabla \cdot\left(\rho_{g} \boldsymbol{f} \boldsymbol{D}_{v} \cdot \nabla \omega_{v}+\rho_{b} \boldsymbol{D}_{b} \nabla X\right)
$$


On the importance of heat and mass transfer coupling for the characterization of hygroscopic insulation materials.

\section{Energy conservation}

$$
\begin{aligned}
& \frac{\partial}{\partial t}\left(\varepsilon_{g}\left(\rho_{v} h_{v}+\rho_{a} h_{a}\right)+\bar{\rho}_{b} \bar{h}_{b}+\varepsilon_{s} \rho_{s} h_{s}\right)+\nabla \cdot\left(\left(\rho_{v} h_{v}+\rho_{a} h_{a}\right) \overline{\boldsymbol{v}}_{g}\right) \\
& =\nabla \cdot\left(\lambda_{e f f} \nabla T+\rho_{g} f \boldsymbol{D}_{v}\left(h_{v} \nabla \omega_{v}+h_{a} \nabla \omega_{a}\right)+h_{b} \rho_{b} \boldsymbol{D}_{b} \nabla X\right)
\end{aligned}
$$

\section{Air conservation}

$$
\frac{\partial\left(\varepsilon_{g} \rho_{a}\right)}{\partial t}+\nabla \cdot\left(\rho_{a} \bar{v}_{g}\right)=\nabla \cdot\left(\rho_{g} f D_{v} \nabla \omega_{a}\right)
$$

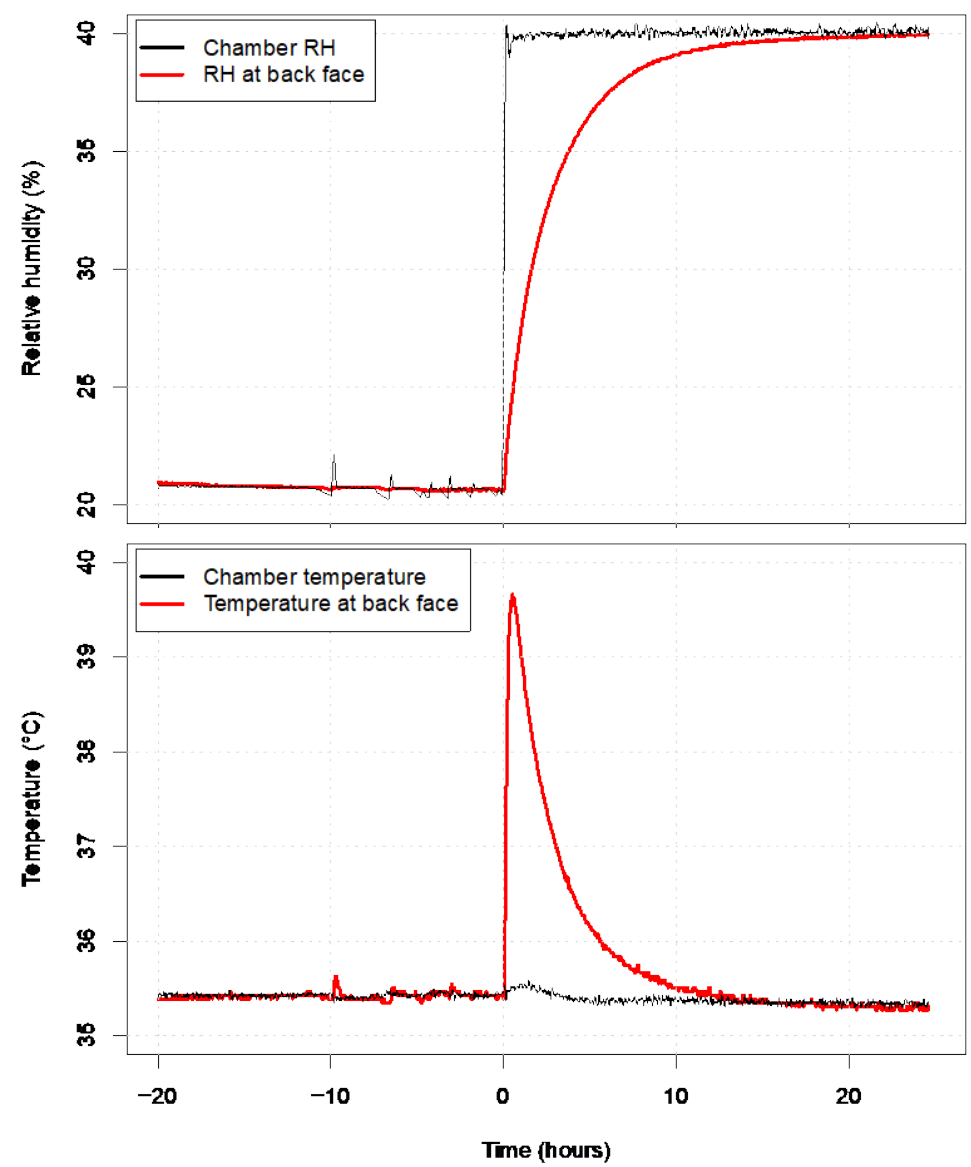

Fig. 2 - Experimental results for a double plate $(2 \times 20 \mathrm{~mm})$ of LDF (160 $\left.\mathrm{kg} . \mathrm{m}^{-3}\right)$ : time evolution of $\mathrm{RH}$ (top) and temperature (bottom) at the back-face of the sample. 


\section{Boundary conditions}

At the external faces of the sample, the boundary conditions were assumed to be of the following form:

$$
\begin{aligned}
& \boldsymbol{J}_{\left.v\right|_{x=0}} \cdot \boldsymbol{n}=h_{m} c M_{v} \ln \left(\frac{1-x_{v, x}}{1-\left.x_{v}\right|_{x=0}}\right) \\
& \boldsymbol{J}_{\left.a\right|_{x=0}} \cdot \boldsymbol{n}=h\left(\left.T\right|_{x=0}-T_{\infty}\right) \\
& P_{\left.g\right|_{x=0^{+}}}=P_{a t m}
\end{aligned}
$$

where $\boldsymbol{J}_{\boldsymbol{v}}$ and $\boldsymbol{J}_{\mathbf{q}}$ represent the fluxes of water vapour and the heat at the boundary, respectively, while $\mathrm{x}$ denotes the distance from the boundary along the external unit normal.

In the previous equations, the barycentric gaseous velocity comes from the generalized Darcy's law:

$$
\overline{\boldsymbol{v}}_{g}=-\frac{\boldsymbol{K} \boldsymbol{k}_{g}}{\mu_{g}} \nabla_{g}
$$

In the hygroscopic domain, the total flux of mass should be expressed as the sum of two parallel fluxes (bound water and water vapour). However, as this work is solely devoted to Low Density Fibreboards, the contribution of bound water diffusion to the macroscopic flux can be neglected. In equations (1-3), $\boldsymbol{D}_{\boldsymbol{v}}$ is the water vapour diffusivity, $\boldsymbol{f}$ the dimensionless diffusivity, $\rho_{g}$ the bulk density of gas, $\rho_{s}$ the bulk density of the lingocellulosic part, $X$ the moisture content (dry basis) and $\omega_{v}$ the mass fraction of water vapour. The reduced diffusivity $\boldsymbol{f}$ characterizes the porous medium: $\boldsymbol{f}$ ranges from 0 (sample impervious to any moisture transfer) to 1 (open sample having the same behaviour of an air layer at rest). This set of equations was solved by the very fast 1 -D version of TransPore with actual boundary conditions used as input data. A full simulation requires about one second of CPU time on a personal computer, even when using thousands of experimental times $t_{i}$ to unroll the boundary conditions. The time step of TransPore was adjusted throughout the simulation, not only to secure convergence, but also to obtain the simulated results at the exact experimental times.

\section{Results and discussion}

Fig. 3 depicts simulation results for our reference test $\left(\boldsymbol{f}=0.5\right.$ and $\left.\lambda=0.05 \mathrm{~W} \cdot \mathrm{m}^{-1} \cdot \mathrm{K}^{-1}\right)$. The $\boldsymbol{f}$ value allows the experimental $\mathrm{RH}$ value at the back-face to match the experimental data. The value of thermal conductivity is determined by a classical mixture law using the gaseous and solid volume fractions and their conductivities. After the sudden increase in $\mathrm{RH}$, water vapour condensates on the front face of the sample. By releasing the latent heat of vaporization, this moisture flux heats up the surface. Because of this temperature 
On the importance of heat and mass transfer coupling for the characterization of hygroscopic insulation materials.

increase, the vapour pressure at the surface increases as well, as it is the product of water activity by the saturated vapour pressure, which increases rapidly with temperature. The primary effect of this change in surface vapour pressure is to reduce the external driving force, which slows down the process ${ }^{[7]}$. This temperature increase eventually affects the whole sample, by a tricky coupling of heat and mass transfer: the temperature increase at one point increases the mass fraction of vapour, which gives rise to a vapour flux that condensates in neighbouring parts. In turn, condensation increases the temperature further inside the medium, which allows the process to continue inwards. This produces a "heat wave" propagating towards the back-face, clearly visible on the temperature profiles. Once this wave reaches the back-face of the sample, a temperature gradient establishes towards the external face, to drive outwards the heat supplied in the medium via the latent heat of vaporization. In figure 3, the effect of this temperature gradient on the mass fraction of vapour is obvious, namely for the profiles at 10 and 30 minutes: although the internal value of moisture content (MC) is still close to the initial value, the mass fraction of vapour already increased significantly due to the temperature rise.
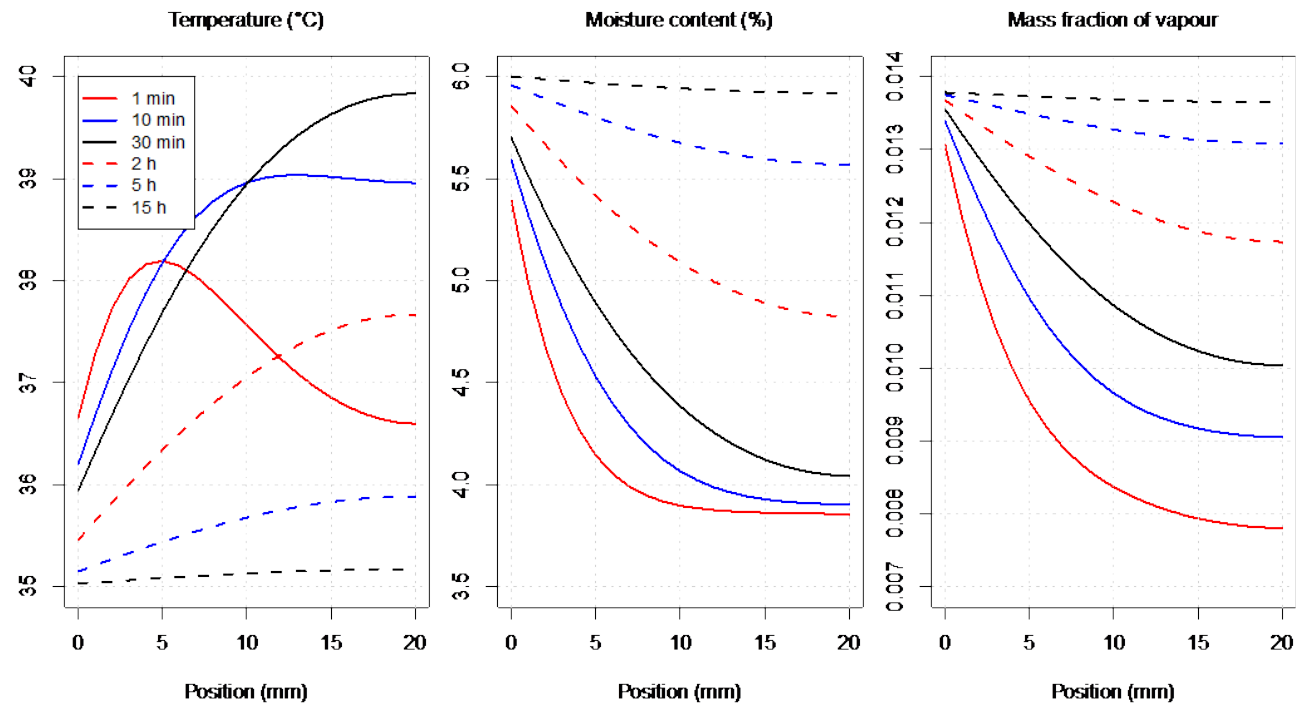

Fig. 3 - Profiles computed at selected times for the reference test $\left(f=0.5\right.$ and $\left.\lambda=0.05 W \cdot \mathrm{m}^{-1} . \mathrm{K}^{-1}\right)$.

The model is also able to compute the time evolution of the two variables measured during the experiment: temperature and $\mathrm{RH}$ at the back face of the sample. The reference test is in perfect agreement with the experiment, which confirms the relevance of the chosen thermal conductivity value. In addition several virtual configurations were computed:

Modified thermal conductivity ( 2 and $\times 0.5$ ): these simulations exhibit the crucial effect of thermal conductivity, not only on the temperature overshoot (peak at respectively $38.6^{\circ} \mathrm{C}$ and $41.2^{\circ} \mathrm{C}$ instead of $39.8^{\circ} \mathrm{C}$ ) but also on the dynamic of $\mathrm{RH}$ evolution, 
No heat and mass coupling: the coupling is easily cancelled by setting the latent heat of vaporization, Lv, to zero. Consistently, the temperature remains at the external temperature. As a consequence of the absence of coupling, the dynamic of $\mathrm{RH}$ evolution is much faster (2.5 hours instead of 4.3 hours for the reference test to attain $35 \%$ of $\mathrm{RH}$ ). This confirms the importance of the heat and mass coupling on the global behaviour.

No internal heat transfer: setting the conductivity $\lambda$ to zero cancels the heat flux inside the sample. Consequently, the latent heat of vaporization released by condensation cannot be driven towards the exchange face. A balance between water intake and temperature rise takes place: the amount of water condensed in the solid phase raises the temperature until the mass fraction of vapour equals the external value. The steady-state is obtained with an MC increase of $0.5 \%$ (against ca. $2 \%$ for the reference test) and an temperature of $43.4^{\circ} \mathrm{C}$. In this extreme, virtual, configuration, the coupling completely stops mass transfer !

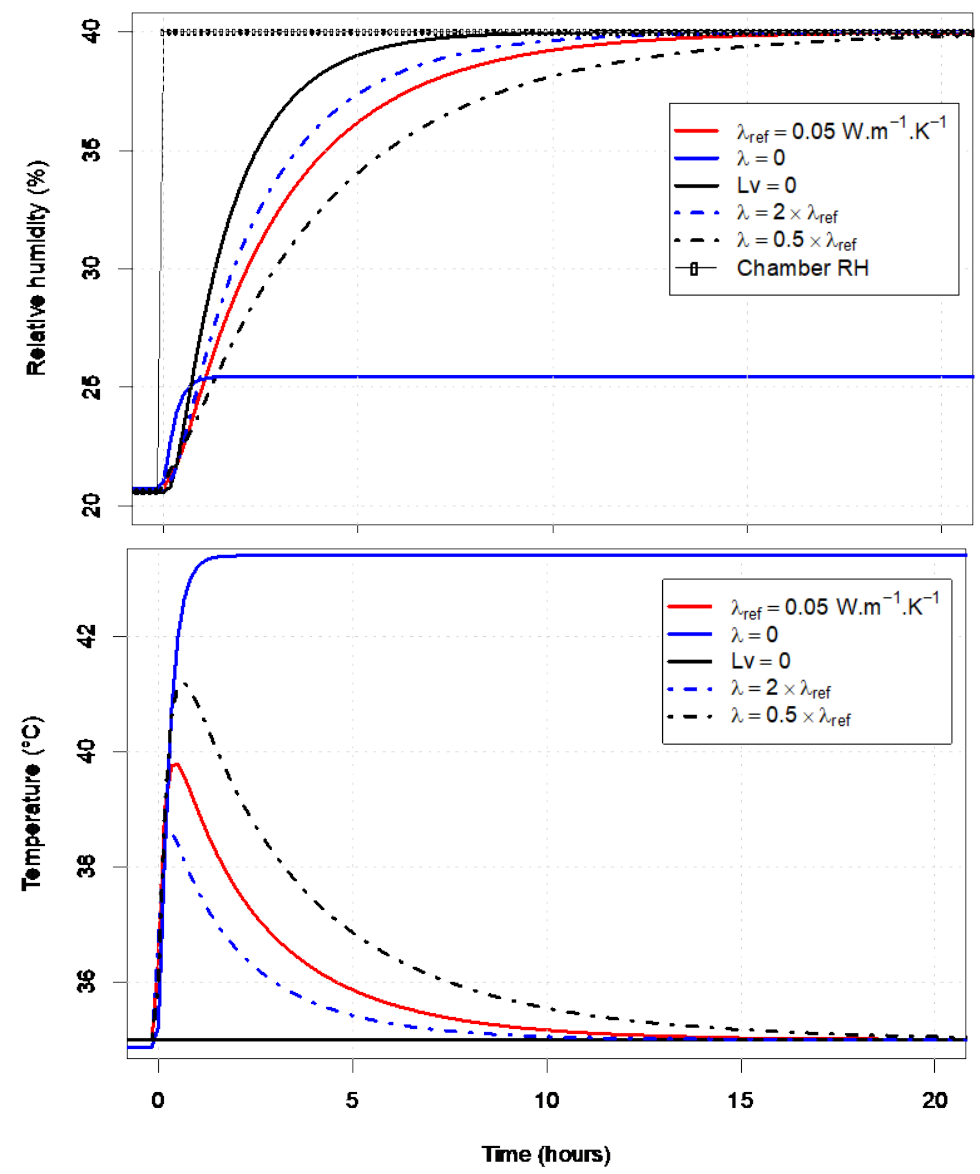

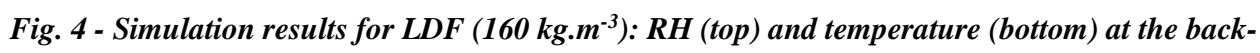
face versus time. The various virtual configurations tested are defined in the legend. 
On the importance of heat and mass transfer coupling for the characterization of hygroscopic insulation materials.

\section{Conclusions and prospects}

Based on experimental data obtained using an original set-up and relevant simulations performed using a comprehensive physical formulation, a throughout vision of the heat mass transfer in Low Density Fiberboards is proposed in the present study. In particular, we emphasize on:

- The impressive change in core temperature in terms of magnitude and duration,

- The great impact of the internal temperature gradient, which slows down mass diffusion,

- $\quad$ The dramatic error on mass diffusivity value if the coupling is ignored.

Further works are in progress in our team to go even further in the analysis of coupled transfers in hygroscopic materials used for building insulation. Besides the heat and mass transfer coupling, sorption hysteresis, dual-scale effects and molecular relaxation have also to be taken into account. However, the strong coupling between these phenomena forces us to be very careful when determining the unknown parameters by inverse analysis. To gain in robustness, several physical parameters will have to be measured during experiments in transient state. By this way, additional parameter values, such as thermal conductivity, could be determined by inverse method.

\section{References}

[1] Delgado, J., Ramos, N. M., Barreira, E., De Freitas, V., 2010. A critical review of hygrothermal models used in porous building materials. Journal of Porous Media 13, 221-234.

[2] Crawley D.B., Lawrie L.K., Winkelmann F.C., Buhl W., Huang Y., Pedersen C. O., Strand R.K., Liesen R.J., Fisher D.E., Witte M.J., Glazer J., 2001 - EnergyPlus : creating a new-generation building energy simulation program, Energy and Buildings, 33 (4): 319-331.

[3] Piot A., Woloszyn M., Brau J., Abele C., 2011 - Experimental wooden frame house for the validation of whole building heat and moisture transfer numerical models, Energy and Buildings, 43 (6): 1322-1328.

[4] Perré P., Pierre F., Casalinho J., Ayouz M., 2015 - Determination of the mass diffusion coefficient based on the relative humidity measured at the back face of the sample during unsteady regimes, Drying Technology, 33: 1068-1075.

[5] Olek W., Perré P. and Weres J., 2011 - Implementation of a relaxation equilibrium term in the convective boundary condition for a better representation of the transient bound water diffusion in wood, Wood Sci. Technol., 45: 677-691.

[6] Perré P., Turner I., 1999 - A 3-D version of TransPore: a comprehensive heat and mass transfer computational model for simulating the drying of porous media, Int. J. Heat Mass Transfer, 42: 4501-4521.

[7] Perré P., 2015 - The proper use of mass diffusion equations in drying modeling: introducing the drying intensity number, Drying Technology, 33: 1949-1962. 